\title{
International Validation of a Nomogram to Predict Recurrence after Resection of Grade 1 and 2 Nonfunctioning Pancreatic Neuroendocrine Tumors
}

\author{
Charlotte M. Heidsma ${ }^{a}$ Stijn van Roessel ${ }^{a} \quad$ Susan van Dieren ${ }^{a} \quad$ Anton F. Engelsman ${ }^{a}$ \\ Oliver Strobel $^{b}$ Markus W. Buechler ${ }^{b}$ Simon Schimmack ${ }^{b} \quad$ Julie Perinel ${ }^{c}$ \\ Mustapha Adham ${ }^{c}$ Vikram Deshpande ${ }^{d} \quad$ Josefine Kjaer $^{\mathrm{e}}$ Olov Norlen $^{\mathrm{e}}$ \\ Anthony J. Gill ${ }^{f}$ Jaswinder S. Samrag Anubhav Mittalg Frederik J.H. Hoogwater ${ }^{\text {h }}$ \\ Florian Primavesi ${ }^{i}$ Stefan Stättneri,j Marc G. Besselink ${ }^{a}$ Casper H.J. van Eijck ${ }^{k}$ \\ E.J.M. Nieveen van Dijkum ${ }^{a}$
}

aDepartment of Surgery, Cancer Center Amsterdam, Amsterdam UMC, University of Amsterdam, Amsterdam, The Netherlands; ${ }^{b}$ Department of General, Visceral and Transplantation Surgery, Heidelberg University Hospital, University of Heidelberg, Heidelberg, Germany; ' Department of Surgery, Centre Hospitalier Universitaire de Lyon, University of Lyon, Lyon, France; 'Department of Pathology, Massachusetts General Hospital, Harvard Medical School, Boston, MA, USA;

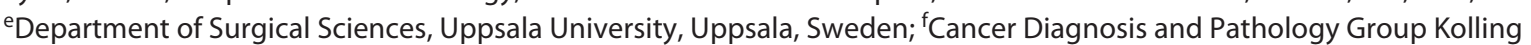
Institute of Medical Research and University of Sydney, Sydney, NSW, Australia; 9Department of Surgery, Royal North Shore Hospital, St Leonards, University of Sydney, Sydney, NSW, Australia; hepartment of Surgery, University Medical Center Groningen, University of Groningen, Groningen, The Netherlands; 'Department of Visceral, Transplant and Thoracic Surgery, Medical University of Innsbruck, Innsbruck, Austria; jDepartment of Surgery, Salzkammergut Klinikum, Standort Vocklabruck, Vocklabruck, Austria; 'Department of Surgery, Erasmus Medical Center, Erasmus University Rotterdam, Rotterdam, The Netherlands

\section{Keywords}

Nonfunctional pancreatic neuroendocrine tumors .

Prediction model · Risk factors · Recurrence

\begin{abstract}
Background: Despite the low recurrence rate of resected nonfunctional pancreatic neuroendocrine tumors (NFpNETs), nearly all patients undergo long-term surveillance. A prediction model for recurrence may help select patients for less intensive surveillance or identify patients for adjuvant therapy. The objective of this study was to assess the external validity of a recently published model predicting recurrence within 5 years after surgery for NF-pNET in an interna-
\end{abstract}

karger@karger.com www.karger.com/nen

Karger $\stackrel{\text { ' }}{5}$

BOPEN ACCESS
(C) 2021 The Author(s)

Published by S. Karger AG, Basel

This is an Open Access article licensed under the Creative Commons Attribution-NonCommercial-4.0 International License (CC BY-NC) (http://www.karger.com/Services/OpenAccessLicense), applicable to the online version of the article only. Usage and distribution for commercial purposes requires written permission. tional cohort. This prediction model includes tumor grade, lymph node status and perineural invasion as predictors. Methods: Retrospectively, data were collected from 7 international referral centers on patients who underwent resection for a grade 1-2 NF-pNET between 1992 and 2018. Model performance was evaluated by calibration statistics, Harrel's C-statistic, and area under the curve (AUC) of the receiver operating characteristic curve for 5 -year recurrencefree survival (RFS). A sub-analysis was performed in pNETs $>2$ $\mathrm{cm}$. The model was improved to stratify patients into 3 risk groups (low, medium, high) for recurrence. Results: Overall, 342 patients were included in the validation cohort with a 5 -year RFS of $83 \%$ (95\% confidence interval [Cl]: 78-88\%). Fifty-eight patients (17\%) developed a recurrence. Calibra- 
tion showed an intercept of 0 and a slope of 0.74 . The C-statistic was 0.77 (95\% Cl: 0.70-0.83), and the AUC for the prediction of 5-year RFS was 0.74 . The prediction model had a better performance in tumors $>2 \mathrm{~cm}$ (C-statistic 0.80$)$. Conclusions: External validity of this prediction model for recurrence after curative surgery for grade 1-2 NF-pNET showed accurate overall performance using 3 easily accessible parameters. This model is available via www.pancreascalculator.com.

(c) 2021 The Author(s).

Published by S. Karger AG, Basel

\section{Introduction}

Pancreatic neuroendocrine tumors (pNETs) are rare neoplasms, accounting for up to $5 \%$ of all pancreatic tumors [1]. The majority of pNETs are nonfunctioning (NF-pNET). Only a small percentage of pNETs cause hormone-induced symptoms (i.e., functioning pNET) [1]. The biological behavior of NF-pNET is very heterogeneous. While some patients present with metastatic disease at initial diagnosis requiring treatment, others present with only a small NF-pNET which can be monitored conservatively with a wait-and-see approach [2]. In general, patients with a solitary NF-pNET have an excellent prognosis. However, this is drastically limited when metastatic spread occurs $[3,4]$. NF-pNET either metastasize locally (remnant pancreas or regional lymph nodes) or distantly (most commonly to the liver) [5]. Different treatment strategies are used to reduce symptoms of metastases and prolong survival. The only curative approach remains complete resection [6].

Following primary tumor resection, different surveillance protocols have been suggested by various international organizations [7-9]. Cross-sectional imaging is recommended in all patients at least once during the first postoperative year. Thereafter, depending on the specific guidelines, imaging is advised every 3-12 months. Currently, surveillance strategies do not clearly differentiate between patients with low or high risk for recurrence. The majority of patients will never develop a recurrence, while they are subjected to excess radiation exposure, unnecessary hospital visits, and related anxiety. Associated healthcare costs could be potentially avoidable. On the other hand, those who do develop recurrent disease could benefit from early detection of metastases and subsequent treatment. Frequently reported risk factors for aggressive behavior in NF-pNET are tumor size, Ki-67 index, tumor differentiation, metastatic lymph nodes, perineural invasion, and lymphovascular invasion $[5,10-13]$. Thus far, none of these risk factors are routinely used to stratify patients into different follow-up groups. Applying various risk factors in a prediction model might aid the stratification of patients for a tailored follow-up strategy.

Recently, 4 prediction models for recurrence after curative resection of pNET have been published $[3,4,12$, $14]$, of which two are not yet validated in an external cohort. The risk model proposed by Genç et al. [3] incorporated 3 readily available variables (i.e., tumor grade, lymph node status, and perineural invasion). These were used to stratify patients with a grade 1-2 NF-pNET into 6 risk groups for 5-year recurrence. The risk model showed good overall performance with a C-statistic of 0.81 , but external validity was not yet assessed. The model is a promising tool to stratify NF-pNET patients due to the high C-statistic. As such, the aim of the current study was to validate this risk score in an international cohort, with an additional validation in NF-pNET $>2 \mathrm{~cm}$. Additionally, the aim was to update the model to be more applicable in a daily setting.

\section{Methods}

\section{Study Design and Patients}

Patients who underwent resection for a non-metastatic grade 1 or 2 NF-pNET were retrospectively identified from institutional databases of 7 international referral centers from 7 countries spanning 3 continents. Participating centers included Royal North Shore Hospital, St. Leonards, Sydney, NSW, Australia; Uppsala University Hospital, Uppsala, Sweden; Heidelberg University Hospital, Germany; Centre Hospitalier Universitaire de Lyon, France; Medical University of Innsbruck, Austria; the University Medical Center Groningen, the Netherlands; and Massachusetts General Hospital, Harvard Medical School, Boston, MA, USA. All included centers are dedicated centers for treatment of patients with NETs and their databases are prospectively maintained.

Patients who underwent standard pancreatoduodenectomy, pylorus-preserving pancreatoduodenectomy, distal pancreatectomy, total pancreatectomy, or enucleation were included. The original model [3] consists of 3 variables: tumor grade (grade 1 or 2), lymph node status (yes/no), and perineural invasion (yes/no). Each outcome was assigned a number of points: grade $1=0$ points, grade $2=40$ points, no positive lymph nodes $=0$ points, positive lymph nodes $=24$ points, no perineural invasion $=0$ points, and perineural invasion $=24$ points. The total sum of points correlated to one of 6 risk percentages for recurrence $(7 \%, 16 \%, 25 \%, 34 \%$, $50 \%, 81 \%)$. The present study grouped these 6 risk groups into 3 groups (low risk $=0-24$ points, medium risk $40-48$ points, high risk $\geq 64$ points) to make the model more clinically applicable.

Patients were excluded from the analysis when they received any type of neoadjuvant or adjuvant treatment, had a genetic syndrome (i.e., multiple endocrine neoplasia type 1 and von HippelLindau syndrome), a functional tumor (i.e., insulinoma, gastrinoma, glucanoma, somatostatinoma, and VIPoma), a grossly positive resection margin (R2), World Health Organization 2017 grade 
Table 1. Patient and tumor characteristics of grade 1 and 2 NF-pNET

\begin{tabular}{|c|c|c|c|}
\hline & $\begin{array}{l}\text { Validation cohort } \\
\text { (present study) } \\
(n=342)\end{array}$ & $\begin{array}{l}\text { Validation cohort }>2 \mathrm{~cm} \\
\text { (present study) } \\
(n=179)\end{array}$ & $\begin{array}{l}\text { Derivation cohort } \\
\text { (Genç et al. [3]) } \\
(n=211)\end{array}$ \\
\hline Age, years, IQR & $60(51-70)$ & $60(48-70)$ & $60(50-66)$ \\
\hline $\mathrm{BMI}, \mathrm{IQR}$ & $25.2(23.0-28.2)$ & $25.8(23.3-28.9)$ & NA \\
\hline Male, $n(\%)$ & $172(50.3)$ & $95(53.1)$ & $103(48.8)$ \\
\hline \multicolumn{4}{|l|}{ ASA, IQR, $n(\%)$} \\
\hline 1 & $21(5.6)$ & $13(7.3)$ & NA \\
\hline 2 & $80(21.4)$ & $27(15.1)$ & NA \\
\hline 3 & $16(4.3)$ & $6(3.4)$ & NA \\
\hline Tumor size, IQR (mm) & $21(12-40)$ & $39(28-50)$ & $25(15-44)$ \\
\hline \multicolumn{4}{|l|}{ Tumor location, $n$ (\%) } \\
\hline Head & $149(46.1)$ & $62(34.6)$ & $80(37.9)$ \\
\hline Body & $106(32.8)$ & $21(11.7)$ & $59(28.0)$ \\
\hline Tail & $62(19.2)$ & $71(39.7)$ & $72(34.1)$ \\
\hline Multiple & $6(1.9)$ & $5(2.8)$ & NA \\
\hline \multicolumn{4}{|l|}{ Tumor grade, $n(\%)$} \\
\hline G1 & $193(56.4)$ & $79(44.1)$ & $139(65.9)$ \\
\hline $\mathrm{G} 2$ & $149(43.6)$ & $100(55.9)$ & $72(34.1)$ \\
\hline \multicolumn{4}{|l|}{ Type of surgery, $n$ (\%) } \\
\hline PD & $68(18.2)$ & $40(22.3)$ & NA \\
\hline $\mathrm{DP}$ & $138(36.9)$ & $68(38.0)$ & NA \\
\hline Central pancreatectomy & $19(5.1)$ & $3(1.7)$ & NA \\
\hline EN & $36(9.6)$ & $6(3.4)$ & NA \\
\hline $\mathrm{TP}$ & $8(2.1)$ & $6(3.4)$ & NA \\
\hline Major complications, $n(\%)$ & $53(15.5)$ & $22(12.6)$ & $58(26.5)$ \\
\hline \multicolumn{4}{|l|}{ Resection margin, $n(\%)$} \\
\hline Ro & $293(89.9)$ & $136(76.0)$ & $179(84.8)$ \\
\hline $\mathrm{R} 1$ & $28(8.6)$ & $21(11.7)$ & $32(15.2)$ \\
\hline Positive lymph nodes, $n$ (\%) & $93(27.2)$ & $71(39.7)$ & $51(24.2)$ \\
\hline Perineural invasion, $n(\%)$ & $74(21.9)$ & $54(30.2)$ & $28(13.3)$ \\
\hline Vascular invasion, $n(\%)$ & $83(29.5)$ & $65(36.3)$ & $50(23.7)$ \\
\hline Mortality, $n(\%)$ & $30(8.8)$ & $22(12.3)$ & $19(9)$ \\
\hline Disease-related & $22(6.4)$ & $16(8.9)$ & $9(4.3)$ \\
\hline Recurrence, $n(\%)$ & $58(17.0)$ & $34(19.0)$ & 35 (16.6) \\
\hline
\end{tabular}

All reported values are in median (IQR, interquartile range) or frequencies (\%, percentage). NF-pNET, nonfunctional pancreatic neuroendocrine tumor; BMI, body mass index; ASA, American Association of Anesthesiologists; G1, grade 1; G2, grade 2; NA, not applicable; PD, pancreatoduodenectomy; DP, distal pancreatectomy; TP, total pancreatectomy; EN, enucleation.

3 tumor (i.e., Ki-67 index >20\%) [15], metastases at the time of surgery (either detected through radiological imaging or histopathologically proven), or missing variables of the nomogram (i.e., tumor grade, lymph node status, perineural invasion status, and recurrence status). Patients with surgery-related mortality, defined as death within 90 days of pancreatic resection, were also excluded. No patients who were in the derivation cohort were included in this study to ensure external validity. The local Institutional Review Board of each participating center either approved the present study or issued a waiver because of the retrospective nature of this study.

\section{Data Collection}

Clinical parameters, pathologic tumor characteristics, and recurrence and survival data were collected locally and provided by each participating center. All pathological specimens were re-examined by an experienced local pathologist who confirmed the diagnosis pNET and determined the tumor size, resection margin, Ki-67 index, and mitotic index (used to determine tumor grade), tumor differentiation, lymph node status, and perineural invasion.

\section{Definitions and Outcome Measures}

Resections were considered margin-negative (R0) if there was no tumor involvement within $1 \mathrm{~mm}$ of each microscopically assessed margin [16]. The original model used the WHO 2010 classification to define tumor grade [17]. In 2017, a new classification was published, where a grade 1 tumor was defined as KI-67 index $\leq 3 \%$ (WHO 2017), instead of $<3 \%$ (WHO 2010) [15]. The model was updated to use the new 2017 classification to define tumor grade. Recurrence was defined as a new local or metastatic lesion, 


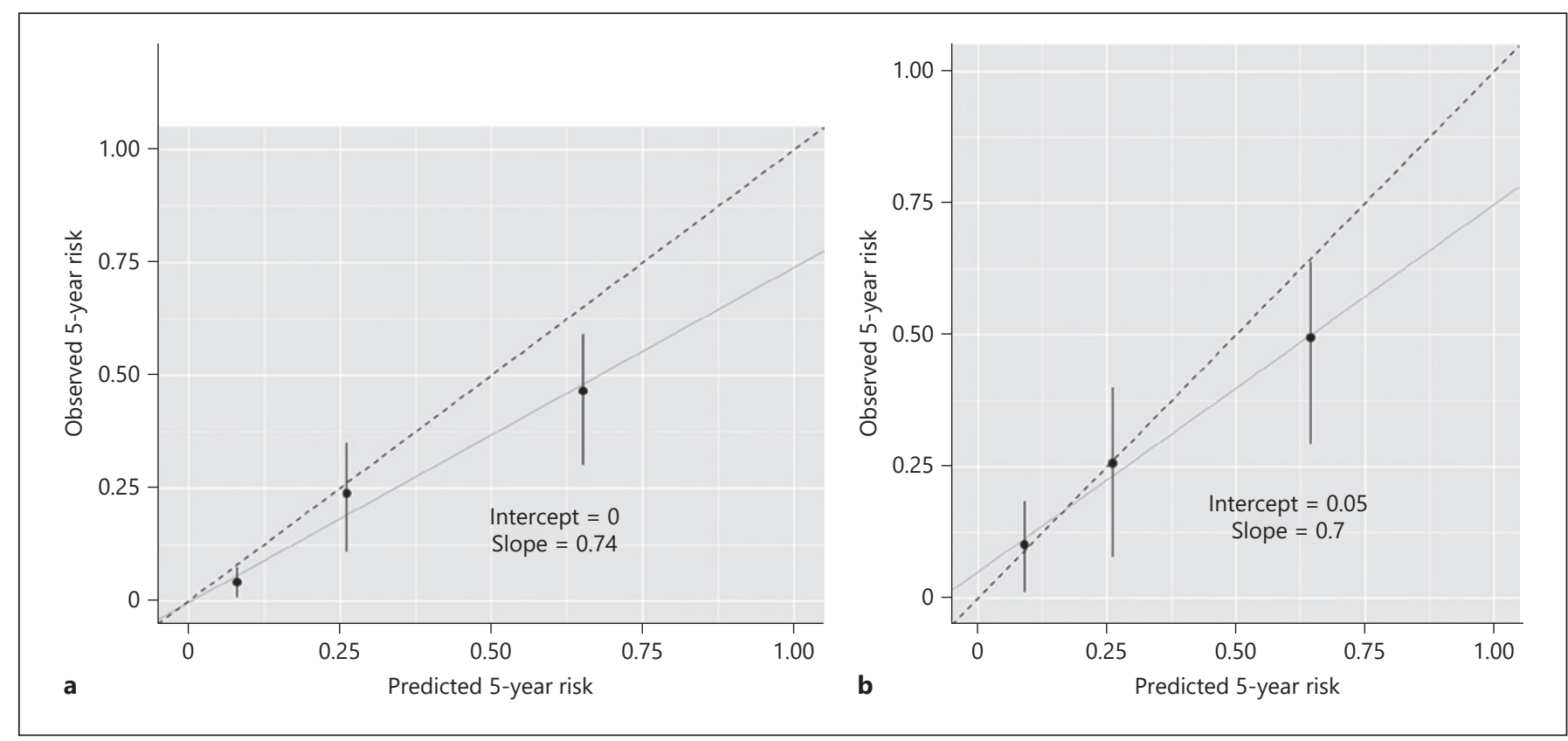

Fig. 1. a Calibration plot of predicted versus observed 5-year risk of recurrence in resected grade 1-2 NF-pNET for the 3 risk groups (low, medium, high) of the prediction model in the validation cohort. The dotted line (slope of 1) represents perfect calibration when combined with an intercept of 0 . $\mathbf{b}$ A calibration plot of pre-

either histologically proven or clinically confirmed by radiological imaging on computed tomography scan, magnetic resonance imaging scan, or gallium-68 dotatate PET scan. The date of recurrence was defined as the date of the first cross-sectional imaging scan on which a new tumor lesion was detected. Tumor size $(\mathrm{cm})$ was determined based on the final histopathology report. Primary outcome for this analysis was 5-year recurrence-free survival (RFS), calculated as time in months from the date of surgery until date of recurrence, or censored at the date of the last follow-up.

\section{Statistical Analysis}

Baseline characteristics were presented as medians with interquartile range (IQR) for continuous nonnormally distributed variables, as means and standard deviation for continuous normally distributed variables, and as frequencies and percentages (\%) for categorical variables. Statistical differences were tested using the $\chi^{2}$ for categorical data, or Student's $t$-test or the Mann-Whitney U test for continuous data. RFS was assessed using the Kaplan-Meier method, presented as median RFS with corresponding 95\% confidence interval (CI), and compared between groups using the logrank test. Two-tailed $p$ values of $<0.05$ were considered statistically significant. All analyses were performed using R version 3.4.3 (cran.r-project.org) and IBM SPSS Statistics for Windows version 24.0 (IBM Corp., Armonk, NY, USA).

In the derivation cohort, 5 risk groups were proposed for 5 -year risk of recurrence. The risk groups were updated in the validation model into 3 risk groups (low risk $=0-24$ points, medium risk $=$ $40-48$ points, high risk $=64-88$ points). Predicted probabilities for 5 -year RFS were calculated for each patient using the baseline sur- dicted versus observed 5-year risk of recurrence in resected grade 1-2 NF-pNET $>2 \mathrm{~cm}$ for the 3 risk groups (low, medium, high) the prediction model in the validation cohort. The dotted line (slope of 1) represents perfect calibration when combined with an intercept of 0 . NF-pNET, nonfunctional pancreatic neuroendocrine tumor. vival function $\left(b=0.853^{\exp (\mathrm{LP})}\right)$ and coefficients (intercept -0.809 ; tumor grade $=-1.403$; positive lymph nodes $=-0.892$; perineural invasion $=-0.867$ ) from the Cox model of the original report [3]. Predicted risk was calculated as 1-RFS. Model performance was tested by calibration and discrimination statistics in the validation cohort. Calibration compares predicted 5-year risk of recurrence with observed risk (using Kaplan-Meier estimates) for different risk groups, with perfect calibration characterized by an intercept of 0 and a slope of 1 . Agreement between observed and predicted probabilities for 5-year recurrence was visualized in a calibration plot and in a table. Discrimination evaluates the model's ability to correctly classify subjects according to their actual outcomes. Harrel's C-statistic was calculated as the overall model performance by the original Cox model (regardless of a specific point in time). A receiver operating characteristics (ROC) curve was construed for the prediction of 5-year RFS specifically, and the area under the curve (AUC) was calculated. The validation of the model was repeated in the same cohort but only in patients with pNET $>2 \mathrm{~cm}$. The validation was reported according to the TRIPOD statement [18]. This model was made available at www.pancreascalculator.com.

\section{Results}

\section{Baseline Characteristics}

In total, 374 patients met the inclusion criteria. Of those, 32 patients $(8.6 \%)$ were excluded due to missing 
data. The final validation cohort consisted of 342 patients. The median age was 60 (IQR 51-70) years, median tumor size was 21 (IQR 13-40) $\mathrm{mm}$, and the majority of patients ( $n=149,46.1 \%)$ had a tumor located in the pancreatic head. Compared to the derivation cohort, there were more patients with grade 2 tumors $(n=149,43.6 \%$ vs. $n$ $=72,34.1 \%)$. In total, 58 events occurred (i.e., NET-related recurrence) during a median follow-up duration of 50.5 (IQR 22.3-103.0) months. Forty-five patients (13.2\%) developed liver metastases only, and 13 patients (3.8\%) developed recurrence in multiple sites (i.e., liver and bone, and liver and lymph nodes). Baseline characteristics of the derivation and validation cohort, including a subset of patients with tumors $>2 \mathrm{~cm}$, are presented in Table 1.

\section{Model Validation}

For the entire cohort, the 5-year RFS was 83.0\% (95\% CI: 78.0-88.0\%), resulting in a 5-year risk of recurrence of $17.0 \%$. Five patients changed from a grade 1 to a grade 2 NF-pNET after the model update to the WHO 2017 tumor grade classification [15]. In the low- and high-risk groups, a less number of patients developed a recurrence as predicted. The predicted 5-year risk of recurrence ranged from $8.1 \%$ (24 points or less) for the low-risk group to $26.1 \%$ (between 40 and 48 points) for the medium-risk group to $65.3 \%$ ( $\geq 64$ points) for the high-risk group. Agreement between predicted and observed 5 -year risk of recurrence is shown in the calibration plot (Fig. 1a) and in Table 2, with an intercept of 0 and a calibration slope of 0.74 . The AUC for prediction of 5-year recurrence was 0.74 (as compared to 0.80 in the derivation cohort). Harrel's C-statistic was 0.77 (95\% CI: 0.710.83 ), as compared to 0.81 (95\% CI: $0.75-0.87$ ) in the derivation cohort. The Kaplan-Meier curve of the 3 risk groups of the total cohort is shown in Figure 2a.

The risk groups had a corresponding 5 -year RFS of 95.8\% (95\% CI: 92.8-99.2), 76.1\% (95\% CI: 65.0-89.1) and $53.4 \%$ (95\% CI: 40.9-69.8) for low, medium, and high-risk patients, respectively. A new nomogram was created to include the 3 risk groups, instead of the original 6 (Fig. 3).

\section{Model Validation for NF-pNET $>2 \mathrm{~cm}$}

In total, 163 patients had an NF-pNET $>2 \mathrm{~cm}$, with a 5 -year RFS of $74.2 \%$ (95\% CI: 66.8-80.8). In these patients, the nomogram predicted lower 5-year risk in all 3 risk groups. Agreement between predicted and observed 5 -year risk of recurrence is shown in the calibration plot (Fig. 1b) and Table 2, with an intercept of 0.05 and a slope

Validation of a Nomogram for Risk of

Recurrence in Resected NF-pNET
Table 2. Predicted versus observed risk of recurrence in grade 1 and 2 NF-pNET

\begin{tabular}{lll}
\hline & $\begin{array}{l}\text { Predicted } \\
\text { 5-year risk, } \%\end{array}$ & $\begin{array}{l}\text { Observed } \\
\text { 5-year risk, \% }\end{array}$ \\
\hline Total cohort & & \\
Low risk $(n=181)$ & 8 & 4 \\
Medium risk $(n=79)$ & 26 & 24 \\
High risk $(n=82)$ & 65 & 47 \\
$>2$ cm cohort & & \\
Low risk $(n=62)$ & 8 & 4 \\
Medium risk $(n=40)$ & 26 & 24 \\
High risk $(n=61)$ & 65 & 49 \\
\hline
\end{tabular}

NF-pNET, nonfunctional pancreatic neuroendocrine tumor.

of 0.7. The AUC for prediction of 5-year recurrence was 0.70 , which was slightly lower than that in the total cohort (0.74) and the derivation cohort (0.80). Harrel's C-statistic was 0.79 (95\% CI: 0.729-0.853) which was better than that in the total cohort and slightly lower than in the derivation cohort.

For an NF-pNET $>2 \mathrm{~cm}$, the risk groups had a 5 -year RFS of $89.8 \%$ (95\% CI: 81.5-98.9), 74.4\% (95\% CI: 60.0 92.2), and 50.6\% (95\% CI: 36.1-70.7) for low-, medium-, and high-risk patients, respectively. The Kaplan-Meier curve of the 3 risk groups of the total cohort is shown in Figure 2b.

\section{Discussion}

This study assessed external validity of a previously published model [3] to predict the risk of recurrence within 5 years after resection of primary nonmetastatic NF-pNET, including the following risk factors: tumor grade, lymph node status, and perineural invasion. Lowrisk patients identified with this nomogram have a predicted 5 -year recurrence risk of $8.1 \%$ versus $26.1 \%$ for medium-risk patients, and $65.1 \%$ for high-risk patients. The model performed well in the external validation cohort, with a C-statistic of 0.77 and an AUC of 0.74. Compared to the ENETS/AJCC eighth staging system for welldifferentiated pNET (C-index 0.76) [19] and the WHO grade classification (C-index 0.76) [15], the current model performed slightly better (C-index 0.77 ).

There is substantial controversy regarding the management of pNET with wide variations among different centers in indications for surgical resection and optimal surveillance regimens $[7,8]$. The heterogenic behavior of 

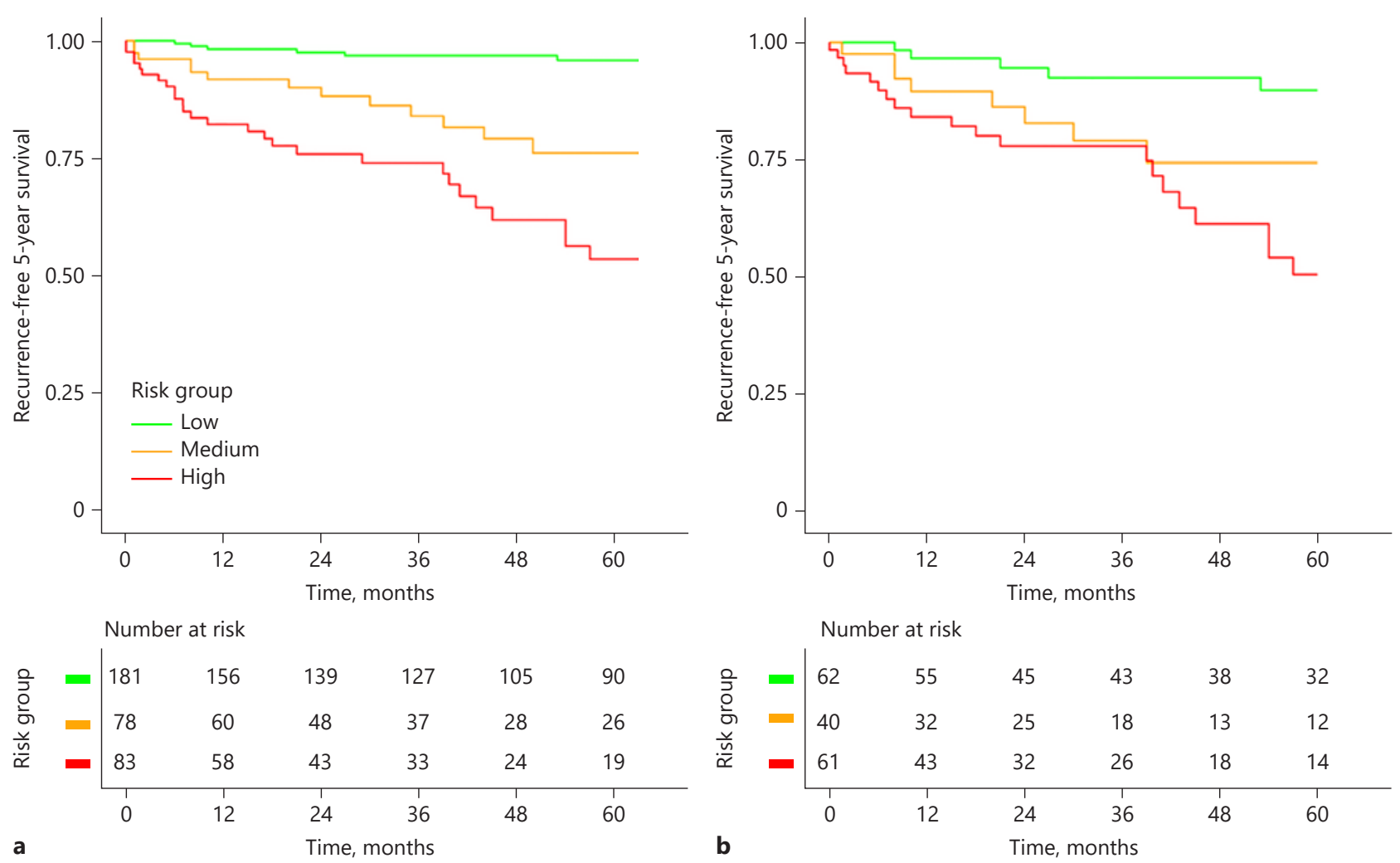

Fig. 2. a Kaplan-Meier curve of the 5-year RFS in resected NFpNET per risk group (low, medium, high) as predicted by the prediction model. b Kaplan-Meier curve of the 5-year RFS in NF-
pNET $>2 \mathrm{~cm}$ per risk group (low, medium, high) as predicted by the prediction model. RFS, recurrence-free survival; NF-pNET, nonfunctional pancreatic neuroendocrine tumor.
Fig. 3. Nomogram to predict 5-year risk of recurrence after resection of a grade 1-2 NF-pNET. NF-pNET, nonfunctional pancreatic neuroendocrine tumor.

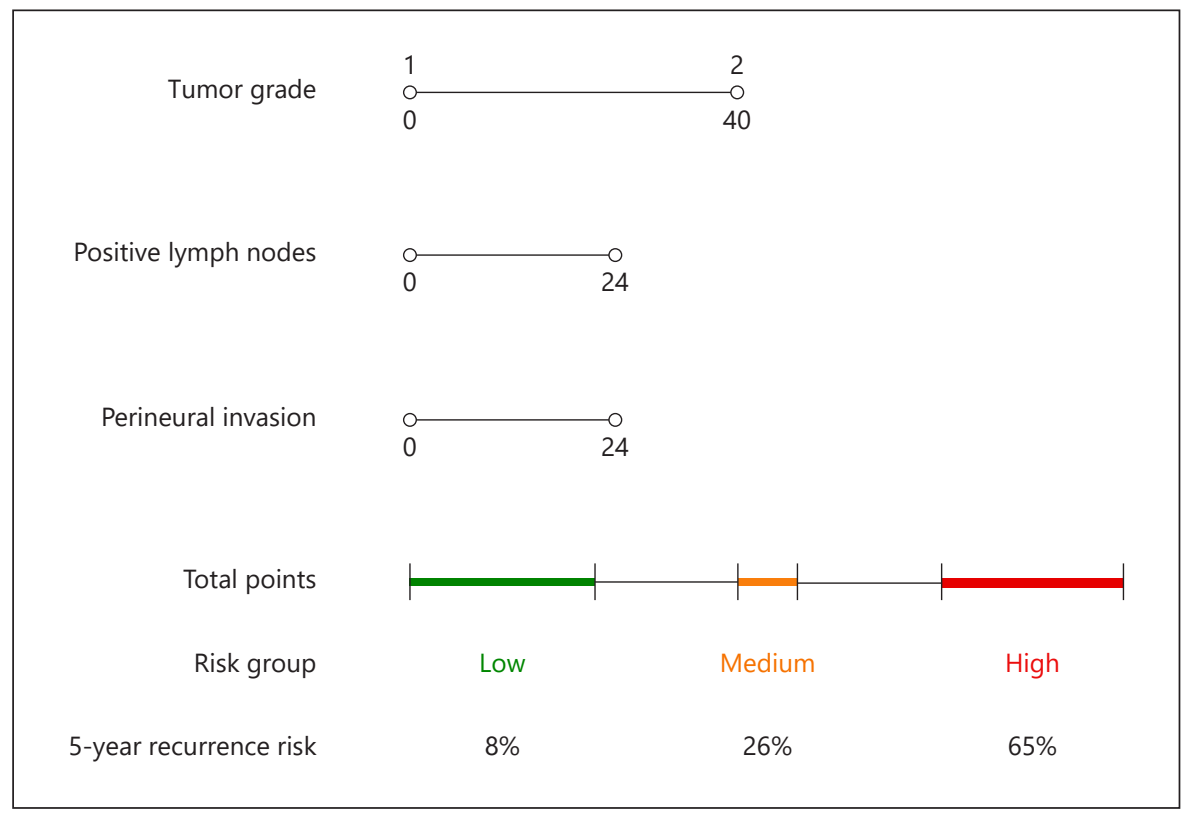


pNET, combined with the increasing availability of treatment options for metastasized disease, make risk stratification vital in these rare tumors. In fact, the majority of patients with a resected pNET will never develop a recurrence [1]. Patient-tailored follow-up regimens are lacking. The use of prediction models could help differentiate between patients who would potentially benefit from more intense follow-up and patients who might not need close surveillance. Since the long-term survival rate of patients after resection of a pNET is often high, they may undergo unnecessary follow-ups for up to 10 years after surgery. Not only may this create misuse of medical resources, it could also expose patients to excessive radiation and cause financial strain on those with limited health-care coverage. For this reason, the current study was important as it assesses external validity of an easyto-use nomogram, with readily available histopathological variables. Based on the results of the present study, we propose that follow-up of low-risk patients can be reduced to a 2-year interval.

In the era of the Ki-67 index and tumor size being two of the most influential factors in determining malignant potential of pNET, the use of tumor grade in the present study may be considered outdated [20]. In fact, some studies suggest that different cutoff values (other than $<3 \%, 3-20 \%,>20 \%$ ) would further improve the predictive ability of Ki-67 [10, 21]. Although the specific Ki-67 index may be a more powerful predictor for recurrence, it needs to be considered that a prediction model should be easy to use. Determining the exact Ki-67 index is not yet common practice in all centers. One of the main challenges after validating a prediction model is subsequent routine implementation of the model in the clinical setting [22]. We hypothesize that including Ki-67 as a continuous factor will complicate the nomogram, and prevent physicians from using it to guide patient management. In addition, we performed a subset analysis in patients with pNET $>2 \mathrm{~cm}$ since current guidelines advise a wait-andsee for patients with pNET $\leq 2 \mathrm{~cm}$ without malignant features $[7,8]$. Previous studies reported favorable outcomes in small pNET, even when treated conservatively [23]. Tumor size not being a significant prognostic factor in the derivation cohort used for developing this nomogram [3]. Still, it was assessed whether the high number of small pNET in this cohort did not affect the validation of the model. In the subgroup analysis of pNET $>2 \mathrm{~cm}$, the model actually performed slightly better with a $\mathrm{C}$-index of 0.79 as compared to a $\mathrm{C}$-index of 0.74 of the overall cohort. It seems as though in this cohort, tumor size is a weaker prognostic factor than tumor grade, lymph node status

Validation of a Nomogram for Risk of Recurrence in Resected NF-pNET and perineural invasion. Perhaps it is necessary to include more than just tumor size in future guidelines that advise on watchful waiting in certain patients with a NF-pNET. Recent studies show promising results on tumor stratification for malignant potential through molecular subtypes which differentiate pNET in alpha and beta cell origins [24]. In addition, although a wait-and-see approach is advised for $\mathrm{pNET} \leq 2 \mathrm{~cm}$, no clear international consensus has been reached, and in some centers, these patients will still be recommended for surgery. This nomogram can be applied in a clinical setting to grade 1 and 2 pNET of any size.

Previously, three other prediction models for recurrence after surgery of a pNET have been developed. The US Neuroendocrine Tumor Study Group created a strong, independently validated model using a large multicenter cohort $(n=754)$ with a C-index of 0.74 , but included all gastroenteropancreatic NETs [14]. Given the abundant variation in metastatic pattern and potential of NETs depending on tumor site, it is therefore less applicable in daily practice to determine surveillance strategies for pNET. A second model was developed by an international group in a cohort of 632 patients with pNET. The risk factors included in this model were number of positive nodes, tumor size, Ki-67 index, and vascular/perineural invasion (either/or) [4]. The C-statistic in the validation cohort was high (0.84). However, the use of number of metastatic nodes and a range in Ki-67 index makes the model less applicable in an out-patient setting. Especially in centers where there is no dedicated NET-team, obtaining sufficient tumor variables to apply this model may be challenging. Lastly, a US and Italian collaboration designed a recurrence risk score in a cohort of 1,006 pNET, including symptomatic tumors, tumor size $>2 \mathrm{~cm}$, and $\mathrm{Ki}-67$ index $(<3 \%, 3-20 \%,>20 \%)$ as variables for the model [12]. In our opinion, patients with a Ki-67 index $>20 \%$ should always undergo stringent follow-up, considering the powerful relationship between tumor cell proliferation and metastatic potential [10].

Prior to recommending any prediction model for standard use in centers treating patients with a pNET, a comparison should be made between all available, externally validated models. This can be done by a systematic review, or even better, through validation of multiple models in a large multi-institutional cohort. Comparative analysis of the different published risk-scores should be performed in a large, multicenter, international cohort, once external validity of all risk-scores has been assessed. In the present study, a comparative analysis could not be performed because of the overlap in patients between this 
validation cohort and the derivation cohort of several of the previously mentioned risk scores. Ideally, through the large ENETS collaborative network, the current model should be validated prospectively and internationally through a multicenter study. Preferably, this multinational study would include an adjuvant treatment protocol for patients with a predicted high recurrence risk $(65 \%$ within 5 years). The necessity of adjuvant treatment in high-risk pNET patients is currently discussed in an ENETS consensus meeting.

The present study has several limitations that need to be considered. First, the retrospective approach may have introduced patient selection bias. Those with missing variables of the nomogram were excluded since we did not re-examine the original histopathological reports. Second, differences exist between centers in surgical conduct and specific histological examination of pNET. Nevertheless, an important strength of this study is its multiinstitutional, international approach, including high-volume hepatopancreatobiliary and NET expert centers from around the globe.

In conclusion, we successfully validated a previously published risk model to predict 5-year recurrence after resection of a nonmetastatic grade 1 or 2 pNET. The model performed well (C-statistic 0.77 ) and is readily available at www.pancreascalculator.com. Since low-risk patients can be easily identified, follow-up intensity after resection could be decreased in these patients. The follow-up can be either reduced from every 6 months to, for example, once every 2 years in low-risk patients, or patients could be discharged from follow-up completely. Importantly, physicians should keep in mind that while some patients find comfort in regular cross-sectional imaging, others experience unnecessary stress and exposure to radiation during follow-up. Further use of the model in a prospective, multicenter study would increase the likelihood of this risk score being used in a daily clinical setting.

\section{Acknowledgements}

Prof. Falconi M., MD; Dr. Partelli S., MD, Dr. Genc C., MD; Dr. Jilesen A.P., MD. Language editing: Dr. T. Mackay, MD.

\section{Statement of Ethics}

The Medical Ethics Review Committee of the Amsterdam University Medical Center, Academic Medical Center, granted exemption from requiring ethics approval of the study protocol (W18_406) considering the use of retrospective data already collected in pre-existing databases.

\section{Conflict of Interest Statement}

The authors have no conflicts of interest to declare.

\section{Funding Sources}

The authors declare they did not receive any funding.

\section{Author Contributions}

All persons named as an author have contributed according to the ICMJE Criteria for Authorship.

\section{Data Availability Statement}

All data analyzed during this study are included in this article. Further inquiries can be directed to the corresponding author.

\section{References}

1 Yao JC, Hassan M, Phan A, Dagohoy C, Leary C, Mares JE, et al. One hundred years after "carcinoid": epidemiology of and prognostic factors for neuroendocrine tumors in 35,825 cases in the United States. J Clin Oncol. 2008;26:3063-72.

2 Franko J, Feng W, Yip L, Genovese E, Moser AJ. Non-functional neuroendocrine carcinoma of the pancreas: incidence, tumor biology, and outcomes in 2,158 patients. J Gastrointest Surg. 2010;14:541-8.

3 Genç CG, Jilesen AP, Partelli S, Falconi M, Muffatti F, van Kemenade FJ, et al. A new scoring system to predict recurrent disease in grade 1 and 2 nonfunctional pancreatic neuroendocrine tumors. Ann Surg. 2018;267:1148-54.

4 Pulvirenti A, Javed AA, Landoni L, Jamieson NB, Chou JF, Miotto M, et al. Multi-institu- tional development and external validation of a nomogram to predict recurrence after curative resection of pancreatic neuroendocrine tumors. Ann Surg. 2019. Epub ahead of print.

5 Zhang XF, Xue F, Dong D-H, Lopez-Aguiar AG, Poultsides G, Makris E, et al. New nodal staging for primary pancreatic neuroendocrine tumors: a multi-institutional and $\mathrm{Na}$ tional Data Analysis. Ann Surg. 2021 Jul 1; 274(1):e28-35.

6 Dong DH, Zhang XF, Lopez-Aguiar AG, Poultsides G, Makris E, Rocha F, et al. Resection of pancreatic neuroendocrine tumors: defining patterns and time course of recurrence. HPB. 2020 Feb;22(2):215-23.

7 Falconi M, Eriksson B, Kaltsas G, Bartsch DK, Capdevila J, Caplin M, et al. ENETS consen- sus guidelines update for the management of patients with functional pancreatic neuroendocrine tumors and non-functional pancreatic neuroendocrine tumors. Neuroendocrinology. 2016;103(2):153-71.

8 Kulke MH, Anthony LB, Bushnell DL, de Herder WW, Goldsmith SJ, Klimstra DS, et al. NANETS treatment guidelines: well-differentiated neuroendocrine tumors of the stomach and pancreas. Pancreas. 2010 Aug;39(6): 735-52.

9 Öberg K, Knigge U, Kwekkeboom D, Perren A; ESMO Guidelines Working Group. Neuroendocrine gastro-entero-pancreatic tumors: ESMO clinical practice guidelines for diagnosis, treatment and follow-up. Ann Oncol. 2012;23(Suppl 7):vii124-30. 
10 Genç CG, Falconi M, Partelli S, Muffatti F, van Eeden S, Doglioni C, et al. Recurrence of pancreatic neuroendocrine tumors and survival predicted by Ki67. Ann Surg Oncol. 2018;25(8):2467-74.

11 Partelli S, Gaujoux S, Boninsegna L, Cherif R, Crippa S, Couvelard A, et al. Pattern and clinical predictors of lymph node involvement in nonfunctioning pancreatic neuroendocrine tumors (NF-PanNETs). JAMA Surg. 2013; 148:932-9.

12 Zaidi MY, Lopez-Aguiar AG, Switchenko JM, Lipscomb J, Andreasi V, Partelli S, et al. A novel validated recurrence risk score to guide a pragmatic surveillance strategy after resection of pancreatic neuroendocrine tumors: an International Study of 1,006 patients. Ann Surg. 2019;270:422-33.

13 Primavesi F, Klieser E, Cardini B, Marsoner K, Froschl U, Thalhammer S, et al. Exploring the surgical landscape of pancreatic neuroendocrine neoplasia in Austria: results from the ASSO pNEN Study Group. Eur J Surg Oncol. 2019;45(2):198-206.

14 Merath K, Bagante F, Beal EW, Lopez-Aguiar AG, Poultsides G, Makris E, et al. Nomogram predicting the risk of recurrence after curative-intent resection of primary non-metastatic gastrointestinal neuroendocrine tumors: an analysis of the U S. Neuroendocrine Tumor Study Group. J Surg Oncol. 2018;117: $868-78$.
15 Lloyd RV, Osamura RY, Klöppel G, Rosai J. WHO classification of tumours of endocrine organs. 4th ed. Lyon: International Agency for Research on Cancer; 2017. p. 355.

16 Campbell F, Foulis A, Verbeke C. Dataset for the histopathological reporting of carcinomas of the pancreas, ampulla of Vater and common bile duct. London: The Royal College of Pathologists; 2010.

17 Bosman FT, Carneiro F, Hruban RH, Theise ND. WHO Classification of Tumours of the Digestive System. 4th ed. Lyon: International Agency for Research on Cancer; 2010.

18 Moons KG, Altman DG, Reitsma JB, Collins GS. New guideline for the reporting of studies developing, validating, or updating a multivariable clinical prediction model: the TRIPOD statement. Adv Anat Pathol. 2015;22(5): 303-5.

19 Luo G, Javed A, Strosberg JR, Jin K, Zhang Y, Liu C, et al. Modified staging classification for pancreatic neuroendocrine tumors on the basis of the American Joint Committee on cancer and European Neuroendocrine Tumor Society systems. J Clin Oncol. 2017;35(3): 274-80.
20 Martin-Perez E, Capdevila J, Castellano D, Jimenez-Fonseca P, Salazar R, BeguiristainGomez A, et al. Prognostic factors and longterm outcome of pancreatic neuroendocrine neoplasms: Ki-67 index shows a greater impact on survival than disease stage. The large experience of the Spanish National Tumor Registry (RGETNE). Neuroendocrinology. 2013;98(2):156-68.

21 Gao SW, Huang CS, Huang XT, Chen LH, Chen W, Cai JP, et al. Ki-67 index of $5 \%$ is better than $2 \%$ in stratifying G1 and G2 of the World Health Organization grading system in pancreatic neuroendocrine tumors. Pancreas. 2019;48(6):795-8.

22 Steyerberg EW, Uno H, Ioannidis JPA, van Calster B; Collaborators. Poor performance of clinical prediction models: the harm of commonly applied methods. J Clin Epidemiol. 2018;98:133-43.

23 Sallinen V, Le Large TY, Galeev S, Kovalenko Z, Tieftrunk E, Araujo R, et al. Surveillance strategy for small asymptomatic non-functional pancreatic neuroendocrine tumors: a systematic review and meta-analysis. HPB. 2017;19(4):310-20.

24 Cejas P, Drier Y, Dreijerink KMA, Brosens LAA, Deshpande V, Epstein CB, et al. Enhancer signatures stratify and predict outcomes of non-functional pancreatic neuroendocrine tumors. Nat Med. 2019;25(8):12605. 